\title{
THE TRACTION PROBLEM FOR INCOMPRESSIBLE MATERIALS
}

\author{
BY
}

Y. H. WAN ${ }^{1}$

\begin{abstract}
The traction problem for incompressible materials is treated as a bifurcation problem, where the applied loads are served as parameters. We take both the variational approach and the classical power series approach. The variational approach provides a natural, unified way of looking at this problem. We obtain a count of the number of equilibria together with the determination of their stability. In addition, it also lays down the foundation for the Signorini-Stoppelli type computations. We find second order sufficient conditions for the existence of power series solutions. As a consequence, the linearization stability follows, and it clarifies in some sense the role played by the linear elasticity in the context of the nonlinear elasticity theory. A systematic way of calculating the power series solution is also presented.
\end{abstract}

1. Introduction. In this paper, we analyze another variation of the basic problem studied in Chillingworth, Marsden and Wan $[\mathbf{2}, \mathbf{3}]$, in which only volumepreserving deformations are admissible.

Let $B \subset R^{3}, 0 \in B$, be an open bounded set with smooth boundary $\partial B$. Set $\mathcal{U}=\left\{\phi \mid \phi: \bar{B} \rightarrow R^{3}\right.$ of Sobolev class $\left.H^{s}, \phi(0)=0\right\}$ with $s>\frac{3}{2}+1$. Denote by $C_{\mathrm{vol}}=\{\phi \in \mathcal{U} \mid J(\phi)=1\}$ the space of volume preserving deformations, where $J(\phi)=\operatorname{det} F, F=D \phi$. The first Piola-Kirchhoff stress tensor $P$ is given by $-J p F^{-T}+\partial W / \partial F\left(F^{-T}=\left(F^{-1}\right)^{T}\right)$, where $W=W(X, C), C=F^{T} F$, stands for a smooth stored energy function, and $p$ is an undetermined hydrostatic pressure. For simplicity in notation, we often drop the variable $X$. Denote by $M_{3}$ the space of all $3 \times 3$ matrices with inner product $\langle A, B\rangle=\operatorname{Trace} A^{T} B, \operatorname{sym}=\left\{E \in M_{3} \mid E^{T}=E\right\}$, skew $=\left\{K \in M_{3} \mid K^{T}=-K\right\}$.

As in $[\mathbf{2}, \mathbf{3}]$, we assume the following conditions throughout this paper: $=0$.

(H1) The undeformed state is stress-free with pressure zero; $p_{0} 1=(\partial W / \partial F)\left(I_{B}\right)$

(H2) $c=4\left(\partial^{2} W / \partial C^{2}\right)\left(I_{B}\right)$ is positive definite on traceless symmetric matrices, i.e. there exists a constant $c_{1}>0$, such that $\langle c(X) E, E\rangle \geq c_{1}|E|^{2}$ for all $E \in$ sym with $\operatorname{tr} E=0$.

The conditions (H1) and (H2) are equivalent to: $W(C)$ has a nondegenerate local minimum at $I$ on the manifold of positive symmetric matrices $C, \operatorname{det} C=1$, with the normalization $p_{0}=0$. Let $W^{*}=W-p_{0}(\operatorname{det} F-1)$, so that $\partial W^{*} / \partial F=$ $\partial W / \partial F-J p_{0} F^{-T}$. Thus, replacing $W$ by $W^{*}$ if necessary, we can always assume that $p_{0}=0$.

Received by the editors July 24,1984 .

1980 Mathematics Subject Classification. Primary 73C50, 73C60.

Key words and phrases. Traction problem, incompressible materials, variational principle, linearization stability, Signorini-Stoppelli Schemes.

${ }^{1}$ Research partially supported by DOE contract DE-AT03-8ZER12097. 
Let $\mathcal{L}=\left\{(b, \tau) \mid b: B \rightarrow R^{3}\right.$ of class $H^{s-2}, \tau: \partial B \rightarrow R^{3}$ of class $H^{s-3 / 2}, \int_{B} b d V+$ $\left.\int_{\partial B} \tau d A=0\right\}$. Given any load $l=(b, \tau) \in \mathcal{L}$, a configuration $\phi \in \mathcal{C}_{\mathrm{vol}}$ is called an equilibrium solution for the load $l$ iff there exists $p \in H^{s-1}$ (and hence unique ${ }^{2}$ such that

$$
\begin{aligned}
-\operatorname{Div} P=b & \text { in } B, \\
P N=\tau & \text { on } \partial B .
\end{aligned}
$$

Here, Div $P=\sum_{j=1}^{3} P_{i j, j}=\sum_{j=1}^{3} \partial P_{i j} / \partial X_{j}$. An equilibrium solution $\phi \in C_{\mathrm{vol}}$ is said to be stable iff $\phi$ is a local minimum of the function $V$ on $C_{\mathrm{vol}}$, where $V(\phi)=\int_{B} W(\phi) d V-\langle l, \phi\rangle,\langle l, \phi\rangle=\int_{B}\langle b, \phi\rangle d V+\int_{\partial B}\langle\tau, \phi\rangle d A$.

Set $\Phi(p, \phi)=(-\operatorname{Div} P, P N)$. Let $S O(3)$, the proper orthogonal group on $R^{3}$, act on $\phi, l$ by composition and on $p$ trivially. Then, $\Phi$ is $S O(3)$-equivariant and $S O(3) I_{B}=S O(3)$ is a set of trivial solutions.

Again, as in $[\mathbf{2}, \mathbf{3}]$, the basic problem is

(T) Describe the equilibrium solutions for loads $\lambda l$, with $\lambda>0$, small, and $l$ near some fixed load $l_{0}$.

Specifically, one needs

(a) to count the number of solutions,

(b) to determine their stabilities.

For general reference on elasticity theory, please see $[\mathbf{5}, \mathbf{8 - 1 0}, \mathbf{1 4}, \mathbf{1 5}]$.

The variational approach in $[\mathbf{2}, \mathbf{3}]$ proves to be very powerful and successful in dealing with such a traction problem for compressible materials. The symmetry group $S O(3)$ plays an important role, and a reduced bifurcation equation is obtained through applying the Liapunov-Schmidt procedure twice. Indeed, old results become unified and many new results are obtained. In $\S 2$, we aim to carry out a similar but more complicated analysis for the incompressible materials. It turns out the loads should be classified into the same types as in $[\mathbf{2}]$. One obtains the same bifurcation diagrams for type 0 and type 1 loads. One also gets the same upper bound for types 2, 3, and 4 loads in "nondegenerate" cases, circles of solutions for parallel loads, and homogeneous solutions for "homogeneous" loads (cf. [1]).

The Signorini Scheme $[\mathbf{1 1}, \mathbf{1 2}, \mathbf{1 5}, \mathbf{1 6}]$ enables us to find power series solutions near identity when the applied load possesses no axis of equilibria. This scheme has been generalized in Marsden and Wan [11]. A perturbation scheme for incompressible materials can be found in Green and Spratt [7]. In the last section, we aim to extend the results in [11] to traction problems for incompressible materials. Again, necessary conditions (i.e. Signorini's compatibility conditions, incompressibility conditions) are obtained by formal expansions. A geometric reformulation of the first order conditions enables us to generalize a result of Tolotti [14]. Motivated by the methods in [3], one can get a second order sufficient condition, via results from $\S 2$. As a consequence, the linearization stability result (cf. $[\mathbf{1 1}, \mathbf{6}])$ follows, and it clarifies in some sense the role played by the linear elasticity in the context of the nonlinear elasticity theory. Based on this second order sufficient condition, we work out a generalized Signorini Scheme for incompressible materials.

As a preparation for $\S \S 2$ and 3 , we conclude this section with a study of the "linearized" problem of our problem (E) in terms of a map $\Phi(p, \phi)$.

\footnotetext{
${ }^{2}$ It suffices to show that $\operatorname{Div}\left(J q F^{-T}\right)=0$ and $\left(J q F^{-T}\right) N=0$ imply $q=0$. For $\operatorname{Div}\left(J F^{-T}\right)=$ 0 , we have $D q=0$ in $B$. Thus $D q=0$ on $B$ and $q=0$ on $\partial B$ imply $q=0$.
} 
Let $\mathcal{Q}=\left\{q: \bar{B} \rightarrow R \mid q \in H^{s-1}\right\}$ and $\mathcal{V}=\{u \in \mathcal{U} \mid \operatorname{div} u=0\}$.

1.1. Definition. Let $L=\mathcal{Q} \times \mathcal{V} \rightarrow \mathcal{L}$ be the linear map defined by

$$
L(q, u)=(-\operatorname{Div}[-q 1+c(e)],[-q 1+c(e)] N),
$$

with $e=\frac{1}{2}\left(\nabla u+\nabla u^{T}\right)$.

1.2. PROPOSITION (CF. [9]). Under assumptions (H1) and (H2) on $W$, we have

(a) $\operatorname{ker} L=\{(0, K X) \mid K \in$ skew $\}$,

(b) $\operatorname{Im} L=\mathcal{L}_{e}=$ the space of all equilibrated loads.

For $l=(b, \tau) \in \mathcal{L},(k(l))_{i j}=\int_{B} b_{i} X_{j} d V+\int_{\partial B} \tau_{i} X_{j} d A$. The load $l$ is said to be equilibrated or $l \in \mathcal{L}_{e}$ iff $k(l) \in \mathrm{sym}$. It is easy to see that $l \in \mathcal{L}_{e}$ iff $\langle l, K X\rangle=0$ for all $K \in$ skew.

1.3. LEMmA. $\langle l, w\rangle=0$ for all $w, \operatorname{tr} \nabla w=0$ iff $l=\left(\begin{array}{c}-\operatorname{Div} q \\ q N\end{array}\right)$ for some scalar function $q\left(\in H^{s-1}\right)$.

ProOF. Suppose $l=\left(\begin{array}{c}-\operatorname{Div}_{q N} q 1 \\ q N\end{array}\right)$ for some $q$. Then

$$
\langle l, w\rangle=\left\langle\left(\begin{array}{c}
-\operatorname{Div} q 1 \\
q N
\end{array}\right), w\right\rangle=\langle q 1, \nabla w\rangle=\langle q, \operatorname{tr} \nabla w\rangle=0 .
$$

On the other hand, assume that $l=\left(\begin{array}{l}b \\ \tau\end{array}\right) .\langle l, w\rangle=0$ for all $w$, with $\operatorname{tr}(\nabla w)=0$. First claim $\tau$ must be normal to $\partial B$, i.e. $\tau=g(X) N$. Indeed choose vector fields $w_{n}$ so that $\operatorname{tr}\left(\nabla w_{n}\right)=0,\left\|w_{n}\right\|_{0} \rightarrow 0$ as $n \rightarrow \infty$, and $\left.w_{n}\right|_{\partial B}=$ tangent component $\tau_{t}$ of $\tau$ on $\partial B$.

From $\int\left\langle b, w_{n}\right\rangle d V+\int\left\langle\tau, w_{n}\right\rangle d A=0, \int\left\langle b, w_{n}\right\rangle d V \rightarrow 0$ as $n \rightarrow \infty$, one has $\int\left\langle\tau, w_{n}\right\rangle d V=\int\left\langle\tau_{t}, \tau_{t}\right\rangle d A=0$. Thus, $\tau_{t}=0$. Next, $(\underset{0}{b+\operatorname{Div} g 1})$ kills all $w$, such that $\operatorname{tr} \nabla w=0$. From Hodge theory, $b+\operatorname{Div} g 1=\nabla \varphi=\operatorname{Div} \varphi 1$, with $\varphi(\partial B)=0$. Consequently, $b=-\operatorname{Div}(g-\varphi) 1$. To finish the proof it suffices to take $q=g-$ $\varphi$. Q.E.D.

ProOF. (a) Clearly, $\operatorname{ker} L \supset\{(0, K X) \mid K \in$ skew $\}$. To see the other inclusion, let $(q, u) \in \operatorname{ker} L$. Thus, $0=\langle L(q, u), u\rangle=\langle-q 1+c(e), \nabla u\rangle=\langle c(e), \nabla u\rangle=\langle c(e), e\rangle$ by divergence theorem and symmetry of $c$. From positive definiteness of $c$ on traceless matrices, $e=\left(\nabla u+\nabla u^{T}\right) / 2=0$. Therefore, $u=K X$ for some $K \in$ skew (cf. Fichera [5]). Now Div $q 1=0$ and $q N=0$ imply $q=0$.

(b) $\langle L(q, u), K X\rangle=\langle-q 1+c(\nabla u), K\rangle=0$ for all $K \in$ skew. Thus, $L(q, u) \in \mathcal{L}_{e}$. On the other hand, let $l \in \mathcal{L}_{e}$. By the Korn second inequality, and the equivalence of the norm $\|\nabla u\|_{0}$ and $\|u\|_{1}$ on $u$ such that $\int u d V=0$, one gets $\langle c(\nabla u), \nabla u\rangle=$ $\langle c(e), e\rangle \geq c_{1}|e|^{2} \geq c_{2}\|u\|_{1}^{2}$ on $u$ such that $\int u_{i, j} d V=\int u_{j, i} d V \int u d V=0$, and $\operatorname{div} u=0$. Therefore, by the Lax-Milgram theorem, there exists $u \in \mathcal{V} \cap \mathcal{U}_{\text {sym }}$ such that $\langle c(\nabla u), \nabla w\rangle=\langle l, w\rangle$ for all $w \in \mathcal{V} \cap \mathcal{U}_{\text {sym }}$. Since $\langle l, K X\rangle=0$ for all $K \in$ skew, $\langle c(\nabla u), \nabla w\rangle=\langle l, w\rangle$ for all $w \in \mathcal{V}$. Hence

$$
\left\langle\left(\begin{array}{c}
-\operatorname{Div} c(\nabla u) \\
c(\nabla u) N
\end{array}\right)-l, w\right\rangle=0 \quad \text { for all } w \in \mathcal{V} .
$$

Therefore, by Lemma 1.3 there exists $q$, such that

$$
\left(\begin{array}{c}
-\operatorname{Div} c(\nabla u) \\
c(\nabla u) N
\end{array}\right)-l=\left(\begin{array}{c}
-\operatorname{Div} q 1 \\
q N
\end{array}\right)
$$


or

$$
l=\left(\begin{array}{c}
-\operatorname{Div}(-q 1+c(\nabla u)) \\
(-q 1+c(\nabla u)) N
\end{array}\right) \cdot \text { Q.E.D. }
$$

2. The variational approach. (A) Existence and stability via a potential on $S O(3)$. We begin our variational approach by giving some preliminary results.

2.1. Proposition (CF. EBIN AND MARSDEN [4]). $C_{\mathrm{vol}}$ is a submanifold of $\mathcal{U}$ with tangent space at $\phi \in \mathcal{C}_{\mathrm{vol}}$ given by $T_{\phi} \mathcal{C}_{\mathrm{vol}}=\left\{u \in \mathcal{U} \mid \operatorname{tr}\left(\nabla u F^{-1}\right)=0\right\}$.

Proof. Consider the smooth map $J: U \rightarrow H^{s-1}$, defined by $J(\phi)=\operatorname{det} F$. Clearly, $J^{-1}(1)=C_{\text {vol }}$. Let $\phi \in C_{\text {vol }}$. Then $D J(u)=\operatorname{tr}\left(F^{-1} \nabla u\right)$. Denote $\mathcal{U}=$ $\mathcal{V} \oplus G=\{u \in \mathcal{U} \mid \operatorname{div} u=0\} \oplus\{\nabla \phi-\phi(0) \mid \phi=0$ on $\partial B\}$, obtained from the Hodge decomposition of vector fields. At $\phi=I_{B}$, $\operatorname{ker} D J=\mathcal{V}$ and $D J \mid G$ is an isomorphism onto (i.e. a Dirichlet problem), $J$ is split surjecive. To obtain split surjectivity at $\phi \in C_{\text {vol }}$, one uses the smooth right translations $R_{\phi}(u)=u \circ \phi$. Hence $\mathcal{U}=T_{\phi} C_{\text {vol }} \oplus\left\{F^{-T} \nabla \phi-F^{-T} \nabla \phi(0)|\phi| \partial B=0\right\}$. Q.E.D.

It is convenient to let Skew $=\{(0, K N) \mid K \in$ skew $\}$ and $\mathcal{L}=\mathcal{L}_{e} \oplus$ skew. Thus, $\mathcal{U}_{\text {sym }}=$ skew $^{\perp}=\left\{u \in U \mid \int u_{i, j} d V=\int u_{j, i} d V\right\}$. Recall from the following lemma from $[2]$

2.2. LEMMA. (a) $\mathcal{U}=\{K X \mid K \in$ skew $\} \oplus \mathcal{U}_{\text {sym }}$.

(b) For some neighborhood $U$ of 0 in $\mathcal{U}_{\text {sym }}$, the map $\rho: S O(3) \times\{I+U\} \rightarrow C$. $\rho(Q, I+u)=Q^{-1}(I+u)$ defines a tubular nbd of $S O(3)$ in $C$.

(c) Skew $=\mathcal{U}_{\text {sym }}^{\perp}$.

For $\mathcal{U}_{\text {sym }} \supset G=\{\nabla \phi-\phi(0) \mid \phi=0$ on $\partial B\}$, it follows easily that

2.3. LEMMA. $\left\{I+\mathcal{U}_{\text {sym }}\right\} \cap \mathcal{C}_{\text {vol }}$ is a submanifold of $I+\mathcal{U}_{\text {sym }}$ near $I$, with tangent space $\mathcal{U}_{\text {sym }} \cap \mathcal{V}=\left\{u \in \mathcal{U}_{\text {sym }} \mid \operatorname{div} u=0\right\}$.

The variational approach (cf. Remark 2.12) starts from the following. For scalar or tensor fields $A_{i}$ on $B$, write $\left\langle A_{1}, A_{2}\right\rangle_{V}=\int_{B}\left\langle A_{1}, A_{2}\right\rangle d V$.

2.4. PROPOSITION. The deformation $\phi \in \mathcal{C}_{\mathrm{vol}}$ is an equilibrium solution with a load $\lambda l$ iff there is a $p$ such that $(p, \phi)$ is a critical point of the function $\tilde{V}$ on $\underline{Q} \times \mathcal{C}$, where $\tilde{V}(p, \phi)=\int W(\phi) d V-\langle\lambda l, \phi\rangle-\langle p, \operatorname{det} F-1\rangle_{V}$.

PROOF. For $D_{\phi}\langle p, \operatorname{det} F-1\rangle_{V}(u)=\left\langle p, J \operatorname{tr}\left(F^{-1} \nabla u\right)\right\rangle_{V}=\left\langle J p F^{-T}, \nabla u\right\rangle_{V}$,

$$
\begin{aligned}
D_{\phi} \tilde{V}(u) & =\left\langle\frac{\partial W}{\partial F}, \nabla u\right\rangle_{V}-\langle\lambda l, u\rangle-\left\langle J p F^{-T}, \nabla u\right\rangle_{V} \\
& =\langle\Phi(p, \phi)-\lambda l, u\rangle \quad \text { (by divergence theorem). }
\end{aligned}
$$

Thus, $D_{p} \tilde{V}=0, D_{\phi} \tilde{V}=0$ iff $\Phi(p, \phi)=\lambda l, \phi \in C_{\text {vol }}$. Q.E.D.

To consider equilibrium solutions near $S O(3)$, by Lemma 2.2(b) one needs to examine the critical points $(Q, p, \phi) \in S O(3) \times \mathcal{Q} \times(I+U)$ of the function

$$
\tilde{V}_{\rho}(Q, p, \phi)=\tilde{V}(p, \rho(Q, \phi))=\int W(\phi) d V-\langle\lambda Q l, \phi\rangle-\langle p, \operatorname{det} F-1\rangle_{V}
$$


2.5. Proposition. $(Q, p, \phi) \in S O(3) \times \mathcal{Q} \times(I+U)$ is a critical point of $\tilde{V}_{\rho}$ on $S O(3) \times \mathcal{Q} \times(I+U)$ iff

(a) $\Phi(p, \phi)=\lambda Q l(\bmod$ Skew $)$,

(b) $J(F)=1$, and

(c) $\langle\lambda W Q l, \phi\rangle=0$ for all $W \in$ skew.

Proof. From the equation $D_{\phi} \tilde{V}_{\rho}(u)=\langle\Phi(p, \phi)-\lambda Q l, u\rangle$, we see that $D_{\phi} \tilde{V}_{\rho}=$ 0 on $U_{\text {sym }}$ iff $\Phi(p, \phi) \equiv \lambda Q l \bmod$ Skew. Clearly $D_{p} \tilde{V}_{\rho}=0$ iff $J(F)=1$. For $D_{Q} \tilde{V}_{\rho}(W Q)=-(\lambda W Q l, \phi\rangle, D_{Q} \tilde{V}_{\rho}=0$ iff $\langle\lambda W Q l, \phi\rangle=0$ for all $W \in$ skew. Q.E.D.

Since, $\left\{I+\mathcal{U}_{\text {sym }}\right\} \cap \mathcal{C}_{\text {vol }}$ is a submanifold near $I$ by Lemma 2.3 , we can linearize the map $\mathcal{Q} \times\left\{I+\mathcal{U}_{\text {sym }}\right\} \cap \mathcal{C} \rightarrow \mathcal{L} \bmod$ Skew,$(p, \phi) \rightarrow \Phi(p, \phi)$ mod Skew, to obtain a linear map $\mathcal{Q} \times \mathcal{U}_{\text {sym }} \cap \mathcal{U} \rightarrow \mathcal{L}$ mod skew, $(q, u) \rightarrow L(q, u)$ mod skew (cf. Definition 1.1). This linear map is an isomorphism by Proposition 1.2. Hence, by the inverse mapping theorem, the equation $\Phi(p, \phi) \equiv \lambda Q l$ mod Skew can be solved uniquely near $(0, I) \in \mathcal{Q} \times(I+U) \cap \mathcal{C}_{\text {vol }}$, provided $\lambda \geq 0$ is small, and $l$ is near $l_{0}$. Let us denote this solution by $\left(P_{Q}(\lambda l), \phi_{Q}(\lambda l)\right)$ or simply by $\left(P_{Q}, \phi_{Q}\right)$ when no confusion may arise.

Now, we are ready to carry out the Liapunov-Schmidt procedure.

2.6. Definition. Define $f: S O(3) \rightarrow R$ by $f(Q)=\tilde{V}_{p}\left(Q, p_{Q}, \phi_{Q}\right)$ for $\lambda \geq 0$ small, and $l$ near $l_{0}$.

The following is a standard corollary of Proposition 2.5.

2.7. CoROllary. $\left(Q, P_{Q}, \phi_{Q}\right)$ is a critical point of $\tilde{V}_{p}$ iff $Q$ is a critical point of $f$ on $S O(3)$.

2.8. Proposition. Assume the elastic tensor $c(X): \mathrm{sym} \rightarrow \operatorname{sym}$ satisfies the condition $(\mathrm{H} 2)$ in $\S 1$. Then, there exists a constant $c_{2}>0$ such that $\langle c(e), e\rangle \geq$ $c_{2}\|D u\|^{2}$ for all $u \in \mathcal{U}_{\mathrm{sym}} \cap \mathcal{V}$, where $e=\frac{1}{2}\left(\nabla u+\nabla u^{T}\right)$.

This proposition follows from the second Korn inequality. By Lemma 2.2(b), one may study $V$ by looking at $V \circ \rho$.

2.9. Proposition. $V_{\rho}$ has a local minimum on $S O(3) \times\left\{I+\mathcal{U}_{\mathrm{sym}}\right\} \cap C_{\mathrm{vol}}$ at $\left(Q, \phi_{Q}\right)$ iff $f$ has a local minimum at $Q$.

ProOF. It suffices to show that, to each $\left(Q, \phi_{Q}\right)$, there exists a neighborhood $\mathcal{U}_{1} \times \mathcal{U}_{2}$ of $\left(Q, \phi_{Q}\right)$ such that $\left.V_{p}\right|_{\tilde{Q} \times \mathcal{U}_{2}}$ has a nondegenerate local minimum at $\phi_{\tilde{Q}}$ on $\left(I+\mathcal{U}_{\text {sym }}\right) \cap C_{\text {vol }}$. Let $\phi_{\tilde{Q}}+u \in\left(I+\mathcal{U}_{\text {sym }}\right) \cap C_{\text {vol }} .1=J\left(\phi_{\tilde{Q}}+u\right)=1+\operatorname{tr}\left(\tilde{F}^{-1} \nabla u\right)+$ $O\left(\|\nabla u\|^{2}\right)$, implies $\operatorname{tr}\left(F^{-1} \nabla u\right)=O\left(\|\nabla u\|^{2}\right) . P_{Q}=0$, when $\lambda l=0$, so $P_{Q}=o(1)$. Thus

$$
\left|\left\langle P_{Q}, \operatorname{tr} \tilde{F}^{-1} \nabla u\right\rangle\right| \leq\left\|P_{Q}\right\|\left\|\operatorname{tr} \tilde{F}^{-1} \nabla u\right\|=o\left(\|D u\|^{2}\right) .
$$

For $u \in \mathcal{U}_{\mathrm{sym}},\langle\partial W / \partial F, \nabla u\rangle_{V}-\langle\lambda \tilde{Q} l, u\rangle=\left\langle P_{Q}, \operatorname{tr} \tilde{F}^{-1} \nabla u\right\rangle$. Thus $\langle\partial W / \partial F, \nabla u\rangle_{V}-$ $\langle\lambda \tilde{Q} l, u\rangle=o\left(\|D u\|^{2}\right)$.

$$
\begin{aligned}
\left.V_{\rho}\right|_{\tilde{Q} \times \mathcal{U}_{2}} & \left(\phi_{\tilde{Q}}+u\right)-\left.V_{\rho}\right|_{\tilde{Q} \times \mathcal{U}_{2}}\left(\phi_{\tilde{Q}}\right) \\
& =\left[\left\langle\frac{\partial W}{\partial F}, \nabla u\right\rangle_{V}-\langle\lambda \tilde{Q} l, u\rangle\right]+\int\left[\frac{1}{2} \frac{\partial^{2} W}{\partial F^{2}}(\nabla u)^{2}+O\left(|\nabla u|^{3}\right)\right] d V \\
& =o\left(\|D u\|^{2}\right)+\int\left[\frac{1}{2} \frac{\partial^{2} W}{\partial F^{2}}(\nabla u)^{2}+O\left(\|\nabla u\|^{3}\right)\right] d V \\
\geq & c\|D u\|^{2}-K\|D u\|^{3}>0
\end{aligned}
$$


for small $\mathcal{U}_{1}, \mathcal{U}_{2}, \lambda$ and $\left\|l-l_{0}\right\|$, by Proposition 2.8 and continuity arguments. Q.E.D. $Q$.

2.10. COROLlary (OF THE PROOF). Index of $V$ at $Q^{-1} \phi_{Q}=$ Index of $f$ at

Summarizing:

2.11. THEOREM. The equilibrium solutions of the incompressible traction problem are in 1-1 correspondence $\left(Q^{-1} \phi_{Q} \leftrightarrow Q\right)$ with the critical points of a function $f$ on $S O(3)$. The stable solutions correspond to local minima of $f$.

2.12. REMARKS. (a) It follows easily from the equation $\langle\partial W / \partial F, \nabla u\rangle_{V}-\langle\lambda l, u\rangle-$ $\left\langle J p F^{-T}, \nabla u\right\rangle_{V}=\langle\Phi(p, \phi)-\lambda l, u\rangle$ that equilibrium solutions are critical points of $V$ on $C_{\text {vol }}$. Indeed, they are the same. To see the converse, we need a variant (or extension) of Lemma 1.3.

(b) Let $\phi \in C_{\text {vol }}$. Then, $\langle l, u\rangle=0$ for all $\operatorname{Tr}\left(F^{-1} \nabla u\right)=0$ iff

$$
l=\left(\begin{array}{c}
-\operatorname{Div} J q F^{-T} \\
J q F^{-T} N
\end{array}\right)
$$

for some function $q$. Proof:

$$
\begin{aligned}
\langle l, u\rangle & =\int b(X) \cdot u(X) d v+\int \tau(X) \cdot u(X) d A \\
& =\int b\left(\phi^{-1} x\right) \cdot u\left(\phi^{-1} x\right) d v+\int \tau\left(\phi^{-1} x\right)\left(\frac{d A}{d a}\right) \cdot u\left(\phi^{-1} x\right) d a .
\end{aligned}
$$

For $\operatorname{div} u\left(\phi^{-1} x\right)=0$ iff $\operatorname{Tr}\left(F^{-1} \nabla u\right)=0$, by Lemma 1.3 ,

$$
\begin{aligned}
& b\left(\phi^{-1} x\right)=-\operatorname{div} q\left(\phi^{-1} x\right)=-F^{-T} \nabla q, \\
& \tau\left(\phi^{-1} x\right)(d A / d a)=q\left(\phi^{-1} x\right) n .
\end{aligned}
$$

Thus, $b(X)=-F^{-T} \nabla q=-\operatorname{Div}\left(J q F^{-T}\right)$ by $\operatorname{Div}\left(J F^{-T}\right)=0$, and $\tau(X)=$ $q(X) n d a / d A=J q F^{-T} N$ by $n d a=J F^{-T} N d A$.

(c) Now, let $\phi \in C_{\text {vol }}$ be a critical point of $V$. Thus

$$
D_{\phi} V(u)=\langle\partial W / \partial F, \nabla u\rangle-\langle\lambda l, u\rangle=\left\langle\left(\begin{array}{c}
-\operatorname{Div} \partial W / \partial F \\
(\partial W / \partial F) N
\end{array}\right)-\lambda l, u\right\rangle=0
$$

on $\operatorname{tr}\left(F^{-1} \nabla u\right)=0$. By the above lemma,

$$
\left(\begin{array}{c}
-\operatorname{Div} \partial W / \partial F \\
(\partial W / \partial F) N
\end{array}\right)-\lambda l=\left(\begin{array}{c}
-\operatorname{Div} J q F^{-T} \\
J q F^{-T} N
\end{array}\right)
$$

for some $q$, or $\Phi(q, \phi)=\lambda l$.

(d) Combining this result with Proposition 2.4, we see immediately that the pressure $p$ is really a Lagrange multiplier for the optimization of $V$ on the manifold $J=1$.

(B) Load classification and a second order potential on $S_{A_{0}}$. As before, we make the expansions

and

$$
\phi_{Q}(\lambda l)=1+\lambda u_{Q}(l)+O\left(\lambda^{2}\right)
$$

$$
P_{Q}(\lambda l)=\lambda q_{Q}(l)+O\left(\lambda^{2}\right)
$$


2.13. LeMmA. $L\left(q_{Q}(l), u_{Q}(l)\right)=(Q l)_{e}$, where $u_{Q}(l) \in \mathcal{U}_{\mathrm{sym}} \cap \mathcal{V}, L$ is as in Definition 3.1, and $(Q l)_{e}$ is the equilibrated part of $Q l$ according to the decomposition $\mathcal{L}=\mathcal{L}_{e} \otimes$ skew.

Proof. By Proposition 2.5(a)

$$
\Phi\left(\lambda q_{Q}(l)+O\left(\lambda^{2}\right), I+\lambda u_{Q}(l)+O\left(\lambda^{2}\right)\right) \equiv \lambda Q l \bmod \text { skew. }
$$

Thus, $L\left(q_{Q}(l), u_{Q}(l)\right)=(\lambda Q l)_{e}$. Q.E.D.

The load classifications enter into this approach through the following

2.14. Proposition. All the critical points of $f$ are near $S_{A_{0}}=\left\{Q \mid k\left(Q l_{0}\right) \in\right.$ $\operatorname{sym}\}$ for $l=l_{0}$ and $\lambda>0$ small.

PROOF. Otherwise, there exists $\lambda_{n} \searrow 0, Q_{n} \rightarrow Q \notin S_{A_{0}}$, by Proposition 3.7(c). $\left(\lambda_{n} W Q_{n} l_{0}, I+\lambda_{n} u_{Q_{n}}\left(l_{0}\right)+O\left(\lambda_{n}^{2}\right)\right)=0$.

Hence, one must have $\left\langle W Q l_{0}, I\right\rangle=\left\langle W, k\left(Q l_{0}\right)\right\rangle=0$ for all $W \in$ skew, which is absurd. Q.E.D.

Since the map $\Phi$ is $S O(3)$-equivariant, applying De Silva's Lemma if necessary, we may assume in what follows that $l_{0} \in \mathcal{L}_{e}$.

Thus, loads are classified into types $0,1, \ldots, 4$ according to the different topological types of the associated $S_{A_{0}}$. The corresponding critical manifolds $S_{A_{0}}$ are 4 points, $S^{1} \cup 2$ points. $R p^{2} \cup 1$ points, $S^{1} \cup S^{1}$ and $S O(3)$ respectively. This classification of loads is the same as that in [2] for the compressible materials.

Now, we shall derive a second order potential on $S_{A_{0}}$ as in $[\mathbf{2}, \mathbf{3}]$.

2.15. LEMMA. $\left\langle Q l, u_{Q}(l)\right\rangle=\left\langle(Q l)_{e}, u_{Q}(l)\right\rangle=\left\langle c\left(\nabla u_{Q}(l)\right), \nabla u_{Q}(l)\right\rangle_{V}$.

PROOF. This follows from the divergence theorem and $\left\langle q_{Q}(l) 1, \nabla u_{Q}(l)_{V}\right\rangle=0$.

2.16. PROPOSITION.

$$
f(Q)=c+\lambda\left[-\left\langle l, Q^{T} 1\right\rangle-\frac{\lambda}{2}\left\langle c\left(\nabla u_{Q}^{0}\right), \nabla u_{Q}^{0}\right\rangle+O\left(\lambda^{2}\right)+O\left(\lambda\left\|l-l_{0}\right\|\right)\right],
$$

where $L\left(q_{Q}, \nabla u_{Q}^{0}\right)=\left(Q l_{0}\right)_{e}, u_{Q}^{0} \in \mathcal{V}$ and $c=\int W(I) d V$.

Proof. Recall that $\phi_{Q}=I+\lambda u_{Q}(l)+O\left(\lambda^{2}\right)$. Thus,

$$
\begin{aligned}
f(Q)= & \int W\left(\phi_{Q}\right) d V-\left\langle\lambda Q l, \phi_{Q}\right\rangle \\
= & {\left[c+\lambda \int \frac{\partial W}{\partial F}(I)\left(u_{Q}(l)\right) d V+\frac{\lambda^{2}}{2} \int \frac{\partial^{2} W}{\partial F^{2}}\left(u_{Q}(l)\right)^{2} d V+O\left(\lambda^{3}\right)\right] } \\
& -\left\langle\lambda Q l, I+\lambda u_{Q}(l)+O\left(\lambda^{2}\right)\right\rangle .
\end{aligned}
$$

Thus, by Lemma 2.15 ,

$$
f(Q)=c+\lambda\left[\left\langle-l, Q^{T} 1\right\rangle-\frac{\lambda}{2}\left\langle c\left(\nabla u_{Q}(l)\right), \nabla u_{Q}(l)\right\rangle+O\left(\lambda^{2}\right)\right] .
$$

For, $\nabla u_{Q}(l)=\nabla u_{Q}\left(l_{0}\right)+O\left(\left|l-l_{0}\right|\right), u_{Q}\left(l_{0}\right)=u_{Q}^{0}+K X$ for some $K \in$ skew,

$$
\left\langle c\left(\nabla u_{Q}(l)\right), \nabla u_{Q}(l)\right\rangle=\left\langle c\left(\nabla u_{Q}^{0}\right), \nabla u_{Q}^{0}\right\rangle+O\left(\left|l-l_{0}\right|\right) .
$$


Hence,

$$
f(Q)=c+\lambda\left[-\left\langle l, Q^{T} 1\right\rangle-\frac{\lambda}{2}\left\langle c\left(\nabla u_{Q}^{0}\right), \nabla u_{Q}^{0}\right\rangle+O\left(\lambda^{2}\right)+O\left(\lambda\left\|l-l_{0}\right\|\right)\right] \text {. Q.E.D. }
$$

2.17. Definition. Let $f^{*}(Q)=(f(Q)-c) / \lambda$ for $\lambda>0$. Thus

$$
f^{*}(Q)=-\left\langle l, Q^{T} 1\right\rangle-\frac{\lambda}{2}\left\langle c\left(\nabla u_{Q}^{0}\right), \nabla u_{Q}^{0}\right\rangle+O\left(\lambda^{2}\right)+O\left(\lambda\left\|l-l_{0}\right\|\right) .
$$

From this formula of $f^{*}$, one is led to examine the function $-\left\langle l_{0}, Q^{T} 1\right\rangle$ on $S O(3)$ (i.e. $\lambda=0$ ).

For convenience, we collect some relevant facts from [2].

2.18. Proposition. (a) $Q$ is a critical point of $-\left\langle l_{0}, Q^{T} 1\right\rangle$ iff $Q \in S_{A_{0}}$.

(b) $S_{A_{0}}$ is a submanifold in $S O(3)$ and $-\left\langle l_{0}, Q^{T} 1\right\rangle$ is nondegenerate on $\left(T_{Q} S_{A_{0}}\right)^{\perp}$ $\subset T_{Q} S O(3)$ with index that of $Q A_{0}-\operatorname{Tr}\left(Q A_{0}\right) 1\left(A_{0}=k\left(l_{0}\right)\right)$.

This proposition suggests that one should carry out another Liapunov-Schmidt reduction.

Thus, take any normal bundle of $S_{A_{0}}$ in $S O(3)$ with fibres orthogonal to $T_{Q} S_{A_{0}}$. Denote by $Q+n(Q), Q \in S_{A_{0}}$, the critical point of $f^{*}$ restricting the fibre through $Q$. Therefore $n(Q)=\lambda y+O\left(\lambda^{2}\right)$, where $y \in T_{Q} S_{A_{0}}^{\perp}$.

2.19. Definition. Set $\tilde{f}(Q)=f^{*}(Q+n(Q))$ for $Q \in S_{A_{0}}$.

2.20. PROPOSITION.

$$
\tilde{f}(Q)=-\left\langle l, Q^{T} 1\right\rangle-\frac{\lambda}{2}\left\langle c\left(\nabla u_{Q}^{0}\right), \nabla u_{Q}^{0}\right\rangle+O\left(\lambda^{2}\right)+O\left(\lambda\left\|l-l_{0}\right\|\right) .
$$

PROOF. It suffices to observe $\left\langle l,(Q+n(Q))^{T} 1\right\rangle=\left\langle l, Q^{T} 1\right\rangle+O\left(\lambda\left|l-l_{0}\right|\right)+O\left(\lambda^{2}\right)$, which follows from the fact $\left\langle l_{0}, y\right\rangle=0$. Q.E.D.

Let us now state a refinement of Theorem 2.11:

2.21. THEOREM. For $\lambda>0$ small and $l$ near $l_{0}$, the solutions to the incompressible traction problem are in 1-1 correspondence to the critical points of $\tilde{f}(Q)$, $Q \in S_{A_{0}}$. Indeed, if $Q$ is a critical point of $\tilde{f}$ on $S_{A_{0}}$, then $(Q+n(Q))^{-1} \phi_{Q+n(Q)}$ is a solution to the traction problem. Furthermore, the index of $V$ at $(Q+n(Q))^{-1} \phi_{Q+n(Q)}$ equals the index of $\left(Q A_{0}-\operatorname{Tr}\left(Q A_{0}\right) 1\right)$ plus the index of $\tilde{f}$ at $Q$.

(C) The nondegenerate cases, parallel loads, and homogeneous loads. Since the reduced bifurcation function given by Proposition 2.16 is basically the same as that in $[\mathbf{3}]$, the same arguments provide the following results.

2.22 THEOREM. For $\lambda>0$ small, $l$ near $l_{0}$ and $l_{0}$ of type 0 , the incompressible traction problem with load $\lambda l$ has exactly 4 solutions, exactly one of them is stable.

2.23. THEOREM. Let $l=l(c)$ depend on $c \in R^{2}$ with $l(0)=l_{0}$ a type 1 load. Under generic conditions, the incompressible traction problem for load $\lambda l(c)$ has the following bifurcation diagram (near $\left.S^{1}\right)$. Furthermore, it is also universal. 


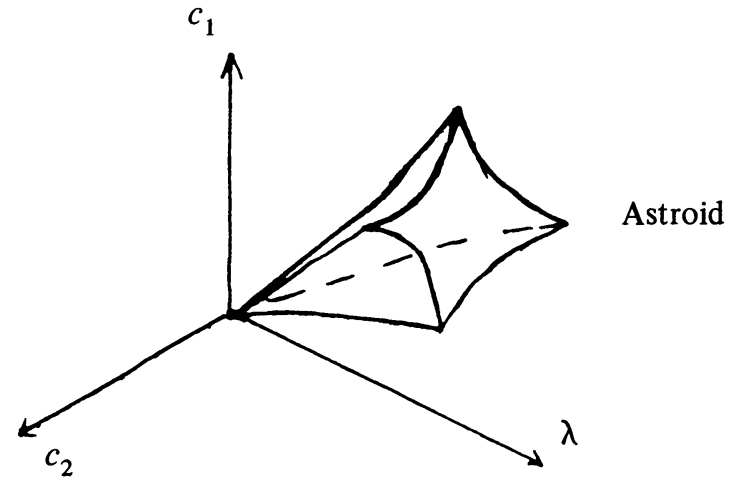

FIGURE 1

2.24. THEOREM. Under generic conditions, the incompressible traction problem for load $\lambda l,\left\|l-l_{0}\right\| / \lambda, \lambda>0$ small, has

(a) at most 13 solutions near $R p^{2}$ for type 2 loads,

(b) at most 8 solutions near $S^{1} \cup S^{1}$ for type 3 loads,

(c) at most 40 solutions near $S O(3)$ for type 4 loads.

Recall that a nontrivial parallel load $l$ is a load in the form $l(X)=f(X) a$, where $f: B \rightarrow R, 0 \neq a \in R^{3}$ and $\int f(X) X d V+\int f(X) X d A \neq 0$. For such a load, the potential function $V$ is $S^{1}$-invariant. Thus, the traction problem becomes degenerate and cannot be covered by previous theorems.

2.25. THEOREM. Let $l_{0}$ be an equilibrated nontrivial parallel load. Then, for $\lambda>0$ small, there exist exactly two circles of equilibrium solutions to our incompressible traction problem. One of them is stable.

A load $l_{0}$ is said to be "homogeneous" if it is in the form $l_{0}=\left(\begin{array}{c}0 \\ A N\end{array}\right)$, with $A \in \operatorname{sym}$. Let us consider the traction problem of an isotropic homogeneous, incompressible material for a homogeneous load. Calculations show the reduced bifurcation equation may always be trivial, and thus one has a degenerate traction problem. We take a direct approach in this case.

2.26. THEOREM. Let $l_{0}$ be a "homogeneous" load. For $\lambda>0$ small, the solution set of a traction problem for an isotropic, homogeneous, incompressible material, consists of homogeneous configurations only and is diffeomorphic to $S_{A_{0}}=$ $\left\{Q \in S O(3) \mid Q A_{0} \in \operatorname{sym}\right\}$.

In other words, no bifurcations occur (cf. [1]). From the representation theorem for the Piola-Kirchhoff stress tensor $P$, one has

2.27. Lemma. $P(p, F)=J(-p+\partial W / \partial F) F^{-T} \in \operatorname{sym}$ for $F \in M$, where $M=\operatorname{sym} \cap\{F \mid \operatorname{det} F=1\}$ is a manifold near $I$.

Proof of ThEOREM 2.26. Let $Q \in S_{A_{0}}=\left\{Q \mid Q A_{0} \in \operatorname{sym}\right\}$, where $A_{0}=$ $k\left(l_{0}\right)=A \operatorname{vol}(B)$. Applying the inverse function theorem to the map $(p, F) \rightarrow$ $P(p, F)$ near $(0, I) \in R^{3} \times M$ one finds $\lambda p_{\lambda Q}, 1+\lambda E_{\lambda Q} \in M$ such that

$$
P\left(\lambda p_{\lambda Q}, 1+\lambda E_{\lambda Q}\right)=\lambda Q A
$$


for $\lambda$ small. Define $\psi_{Q}(X)=Q^{-1}\left(X+\lambda E_{\lambda Q} X\right)$. Clearly $\psi_{Q}(X)$ is homogeneous. $P\left(\lambda P_{\lambda Q}, Q^{-1}\left(1+\lambda E_{\lambda Q}\right)\right)=Q^{-1} P\left(\lambda P_{\lambda Q}, 1+\lambda E_{\lambda Q}\right)=Q^{-1}(\lambda Q A)=\lambda A$. Hence, $-\operatorname{Div} P=0, P N=\lambda A N$ and $\psi_{Q}$ solves the traction problem. Indeed $Q \rightarrow \psi_{Q}$ is a diffeomorphism. By Theorem 2.21,

$$
\begin{aligned}
\left\{\psi \mid Q \in S_{A_{0}}\right\} & \subseteq\{\text { all solutions near } S O(3)\} \\
& \subseteq\left\{(Q+n(Q))^{-1} \phi_{Q+n(Q)} \mid Q \in S_{A_{0}}\right\} \approx S_{A_{0}} .
\end{aligned}
$$

The proof is now completed by topological considerations (i.e. invariance of domain, connectedness). Q.E.D.

3. A power series approach. In this section, let us consider the problem: Given $l(\lambda) \in \mathcal{L}, l(\lambda) \rightarrow 0$ as $\lambda \rightarrow 0$, under what conditions does there exist $\phi(\lambda) \in$ $C_{\mathrm{vol}}, p(\lambda) \rightarrow 0$ and $\phi(\lambda) \rightarrow I$ as $\lambda \rightarrow 0$, such that $\Phi(p(\lambda), \phi(\lambda))=l(\lambda)$ ? If they exist, how do we find them? Here and what follows, $l(\lambda), p(\lambda)$ and $\phi(\lambda)$ are smooth functions in $\lambda$. Let us write $l(\lambda)=\lambda l_{1}+\lambda^{2} l_{2}+\cdots$ (more precisely, $l(\lambda)=\lambda l_{1}+\lambda^{2} l_{2}+\cdots+\lambda^{n} l_{n}+O\left(\lambda^{n+1}\right)$ for any $\left.n\right) \cdot p(\lambda)=\lambda p_{1}+\lambda^{2} p_{2}+\cdots$ and $\phi(\lambda)=I+\lambda u_{1}+\lambda^{2} u_{2}+\cdots$. Throughout this section, $l(\lambda)$ is assumed to be given.

(A) Necessary conditions. Let $(p(\lambda), \phi(\lambda))$ be a solution to the above problem.

3.1. Signorini's compatibility conditions. From Proposition $2.4\left\langle l(\lambda), W^{T} \phi(\lambda)\right\rangle=$ $\langle W l(\lambda), \phi(\lambda)\rangle=0$ for all $W \in$ skew. Thus, $k(l(\lambda), \phi(\lambda)) \in$ sym or

$$
2 \operatorname{skew}\left(k(l(\lambda), \phi(\lambda)) \approx \int l(\lambda) \times \phi(\lambda)=0\right.
$$

where " $\times$ " denotes the usual cross product in $R^{3}$, and $\int l \times \phi=\int_{B} b \times \phi d V+$ $\int_{\partial B} \tau \times \phi d A$. From the equation $\int l(\lambda) \times \phi(\lambda)=0$, we obtain:

$\left(\mathrm{C}_{n-1}\right) \quad \lambda^{n}$ order, $\quad \int l_{1} \times u_{n-1}+\int l_{2} \times u_{n-2}+\cdots \int l_{n} \times I=0$.

3.2. Incompressibility conditions. Since $1=J\left(I+\lambda \nabla u_{1}+\lambda^{2} \nabla u_{2}+\cdots\right)$ by looking at $\lambda^{n}$ order terms, we have:

(I $\left.\mathrm{I}_{1}\right) \quad \lambda^{1}$ order, $\operatorname{tr}\left(\nabla u_{1}\right)=0$,

$\left(\mathrm{I}_{2}\right) \quad \lambda^{2}$ order, $\operatorname{tr} \nabla u_{2}-\frac{1}{2} \operatorname{tr}\left(\nabla u_{1} \nabla u_{1}\right)=0$,

$\left(\mathrm{I}_{n}\right) \quad \lambda^{n}$ order, $\operatorname{tr} \nabla u_{n}-\operatorname{tr}\left(\nabla u_{1} \nabla u_{n-1}\right)+\not\left(\nabla u_{1}, \ldots, \nabla u_{n-2}\right)=0$,

where $\nVdash$ is a polynomial in $\nabla u_{1}, \ldots, \nabla u_{n-2}$ of degree $n\left(\operatorname{deg} \nabla u_{i}=i\right)$.

3.3. Linear equations. Expand $\Phi=\Phi_{1}+\Phi_{2}+\Phi_{3}+\cdots$ near $(0, I)$ as a (formal) Taylor series with $\Phi_{1}=L, \Phi_{2}=\Phi_{2}((q, u),(q, u))$, etc.

Hence,

$$
\begin{aligned}
\Phi\left(\lambda p_{1}\right. & \left.+\lambda^{2} p_{2}+\cdots, I+\lambda u_{1}+\lambda^{2} u_{2}+\cdots\right) \\
= & L\left(\lambda p_{1}+\lambda^{2} p_{2}+\cdots, \lambda u_{1}+\lambda^{2} u_{2}+\cdots\right) \\
& +\Phi_{2}\left(\lambda p_{1}+\lambda^{2} p_{2}+\cdots, \lambda u_{1}+\lambda^{2} u_{2}+\cdots\right)+\cdots \\
= & \lambda l_{1}+\lambda^{2} l_{2}+\cdots
\end{aligned}
$$


Comparing orders in $\lambda$, we get

$\lambda^{1}$ order, $L\left(p_{1}, u_{1}\right)=l_{1}$,

$\left(\mathrm{L}_{n}\right)$

$\lambda^{2}$ order, $L\left(p_{2}, u_{2}\right)+\Phi_{2}\left(p_{1}, u_{1}\right)^{2}=l_{2}$,

$$
\begin{aligned}
\lambda^{n} \text { order, } \quad L\left(p_{n}, u_{n}\right)+ & 2 \Phi_{2}\left(\left(p_{1}, u_{1}\right),\left(p_{n-1}, u_{n-1}\right)\right) \\
& +K\left(\left(p_{1}, u_{1}\right), \ldots,\left(p_{n-2}, u_{n-2}\right)\right)=l_{n}
\end{aligned}
$$

where $\mathcal{K}$ is a polynomial in $\left(p_{1}, u_{1}\right), \ldots,\left(p_{n-2}, u_{n-2}\right)$.

Now, we will reformulate the 1st order necessary conditions in geometric terms.

Recall, $S_{A}=\{Q \in S O(3) \mid k(Q l) \in \operatorname{sym}\}$ for $k(l)=A . T_{Q} S_{A}=\{K Q \mid K k(Q l)=$ $k(K Q l) \in \operatorname{sym}\}$, the tangent space of $S_{A}$ at $Q$. For $k(Q l) \in \operatorname{sym}, L\left(p_{Q}(l), u_{Q}(l)\right)=$ Ql. $u_{Q}(l) \in \mathcal{U}_{\text {sym }} \cap \mathcal{V}$.

3.4. LEMMA. $2\left\langle l_{2}, Q^{T} X\right\rangle+\left\langle c\left(\nabla u_{Q}\left(l_{1}\right)\right), \nabla u_{Q}\left(l_{1}\right)\right\rangle$ restricted to $S_{A_{1}}$ has a critical point $Q \in S_{A_{1}}$ iff $0=\left\langle l_{2},(K Q)^{T} X\right\rangle+\left\langle c\left(\nabla u_{Q}\left(l_{1}\right)\right), \nabla u_{K Q}\left(l_{1}\right)\right\rangle$ for all $K Q \in T_{Q} S_{A_{1}}$.

3.5. Lemma. Let $A \in$ sym. Then, $\langle K A, W\rangle=\langle K, W A\rangle$ is a symmetric bilinear form with kernel $\{K \in \mathrm{skew} \mid K A \in \mathrm{sym}\}$.

The proofs of these two lemmas are elementary and we leave them to the readers.

3.6. THEOREM. Let $l_{1} \in \mathcal{L}_{e}$ (i.e. condition $\left(\mathrm{C}_{0}\right)$ holds). There exist $p_{1}, u_{1}$, $L\left(p_{1}, u_{1}\right)=l_{1}$, with $\operatorname{tr} \nabla u_{1}=0$. $\int l_{1} \times u_{1}+\int l_{2} \times I=0$ iff $2\left\langle l_{2}, Q^{T} X\right\rangle+$ $\left\langle c\left(\nabla u_{Q}\left(l_{1}\right)\right), \nabla u_{Q}\left(l_{1}\right)\right\rangle$ restricted to $S_{A_{1}}$ has a critical point $Q \in S_{A_{1}}$.

PROOF. For $\operatorname{tr} \nabla u_{I}=0$ by the divergence theorem,

$$
\begin{aligned}
& \left\langle c\left(\nabla u_{I}\left(l_{1}\right)\right), \nabla u_{W}\left(l_{1}\right)\right\rangle=\left\langle c\left(\nabla u_{W}\left(l_{1}\right)\right), \nabla u_{I}\left(l_{1}\right)\right\rangle \\
& \quad=\left\langle-q_{W}\left(l_{1}\right)+c\left(\nabla u_{W}\left(l_{1}\right)\right), \nabla u_{I}\left(l_{1}\right)\right\rangle=\left\langle W l_{1}, u_{I}\left(l_{1}\right)\right\rangle .
\end{aligned}
$$

The "only iff" part: $\int l_{1} \times u_{1}+\int l_{2} \times I=0$ implies $\left\langle W l_{1}, u_{1}\right\rangle+\left\langle W l_{2}, I\right\rangle=0$ for all $W \in$ skew, and $u_{I}=u_{1}+K X$ for some $K \in$ skew.

$$
\left\langle W l_{1}, u_{I}\right\rangle=\left\langle W l_{1}, u_{1}\right\rangle+\left\langle W l_{1}, K X\right\rangle=\left\langle W l_{1}, u_{1}\right\rangle+\left\langle K, k\left(W l_{1}\right)\right\rangle .
$$

Thus, $\left\langle l_{2}, W^{T} X\right\rangle+\left\langle c\left(\nabla u_{I}\left(l_{1}\right)\right), \nabla u_{W}\left(l_{1}\right)\right\rangle=\left(\left\langle w l_{2}, I\right\rangle+\left\langle W l_{1}, u_{1}\right\rangle\right)+\left\langle K, k\left(W l_{1}\right)\right\rangle=0$ for $W l_{1} \in$ sym, $W \in$ skew.

By Lemma 3.4, the proof of the "only if" part is now completed.

The "if" part: one needs to find $K \in$ skew so that, for $u_{1}=u_{I}-K X,\left\langle W l_{1}, u_{1}\right\rangle+$ $\left\langle W l_{2}, I\right\rangle=0$ for all $W \in$ skew.

$\left\langle W l_{1}, u_{I}\right\rangle+\left\langle l_{2}, W^{T} X\right\rangle=0$ for $W A_{1} \in \operatorname{sym}\left(\right.$ for $\left\langle c\left(\nabla u_{I}\left(l_{1}\right)\right), \nabla u_{W}\left(l_{1}\right)\right\rangle=$ $\left.\left\langle W l_{1}, u_{I}\right\rangle\right)$ by hypothesis.

By Lemma 3.5 there exists $K \in$ skew such that $\left\langle W l_{1}, u_{I}\right\rangle+\left\langle l_{2}, W^{T} X\right\rangle=$ $\left\langle W A_{1}, K\right\rangle$ for all $W \in$ skew.

Therefore,

$$
\begin{aligned}
\left\langle W l_{1}, u_{1}\right\rangle+\left\langle W l_{2}, I\right\rangle & =\left\langle W l_{1}, u_{I}\right\rangle-\left\langle W l_{1}, K X\right\rangle+\left\langle W l_{2}, I\right\rangle \\
& =\left\langle W l_{1}, u_{I}\right\rangle+\left\langle l_{2}, W^{T} X\right\rangle-\left\langle W A_{1}, K\right\rangle=0
\end{aligned}
$$

for all $W \in$ skew. Q.E.D. 
3.7. COROLlary (EXTENSION OF TOLOTTI $[\mathbf{1 4}]$ ). There exist at least 4 rotations $Q$ in $S O(3)$ such that conditions $\left(\mathrm{C}_{0}\right),\left(\mathrm{C}_{1}\right),\left(\mathrm{L}_{1}\right)$ and $\left(\mathrm{I}_{1}\right)$ hold for $Q l_{1}=l_{1}^{*}$ and $Q l_{2}=l_{2}^{*}$, i.e. $l_{1}^{*} \in \mathcal{L}_{e}$ and $\int l_{1}^{*} \times u_{1}+\int l_{2}^{*} \times I=0$ for some $\left(p_{1}, u_{1}\right)$ with $L\left(p_{1}, u_{1}\right)=l_{1}^{*}, \operatorname{tr} \nabla u_{1}=0$.

ProOF. Let $Q$ be a critical point of $2\left\langle l_{2}, Q^{T} X\right\rangle+\left\langle c\left(\nabla u_{Q}\left(l_{1}\right)\right), \nabla u_{Q}(l)\right\rangle$ on $S_{A_{1}}$.

By Lemma 3.4, $\left\langle l_{2},(K Q)^{T} X\right\rangle+\left\langle c\left(\nabla u_{Q}\left(l_{1}\right)\right), \nabla u_{K Q}\left(l_{1}\right)\right\rangle=0$ for all $K k\left(Q l_{1}\right) \in$ sym.

Since, $u_{K Q}\left(l_{1}\right)=u_{K}\left(Q l_{1}\right)=u_{K}\left(l_{1}^{*}\right)$,

$$
\begin{gathered}
u_{Q}\left(l_{1}\right)=u_{I}\left(Q l_{1}\right)=u_{I}\left(l_{1}^{*}\right), \\
\left\langle l_{2}^{*}, K^{T} X\right\rangle+\left\langle c\left(\nabla u_{I}\left(l_{1}^{*}\right)\right), \nabla u_{K}\left(l_{1}^{*}\right)\right\rangle=0 \text { for all } K k\left(l_{1}^{*}\right) \in \text { sym. }
\end{gathered}
$$

By Theorem 3.6 and Lemma 3.4 (with $Q=I, l_{1}=l_{1}^{*}, l_{2}=l_{2}^{*}$ ), the corollary follows by observing at least 4 such critical points can be found. Q.E.D.

(B) A first order sufficient condition, and a Signorini Scheme. Let $l_{1} \in \mathcal{L}_{e}$ (i.e. an equilibrated load). The load $l_{1}$ has an axis $a \in R^{3},\|a\|=1$, of equilibria if $R_{\theta} l_{1} \in \mathcal{L}_{e}$ for any rotation $R_{\theta}$ with axis $a$. It can be shown that $l_{1}$ has no axis of equilibria iff the function $\left\langle l_{1}, Q^{T} I\right\rangle$ on $S O(3)$ has a nondegenerate critical point at $Q=I$ (cf. [2]).

3.8. ThEOREM. Suppose $l^{\prime}(0)=l_{1} \in \mathcal{L}_{e}$ has no axis of equilibria. Then there exist unique $p(\lambda), \phi(\lambda) \in C_{\text {vol }}$ such that $\Phi(p(\lambda), \phi(\lambda))=l(\lambda), p(\lambda) \rightarrow 0, \phi(\lambda) \rightarrow I$, as $\lambda \rightarrow 0$.

PROOF. It suffices to observe $f=$ constant $-\lambda\left\langle l_{1}, Q^{T} I\right\rangle+O\left(\lambda^{2}\right)$, and $\left\langle l_{1}, Q^{T} I\right\rangle$ has a nondegenerate critical point on $S O(3)$ at $Q=I$. Q.E.D.

It is convenient to state and prove the following lemmas in which $l_{1} \in \mathcal{L}_{e}$ may possess axis of equilibria. These lemmas will also be used in $(C)$.

3.9. Lemma. Let $K \in$ skew. Then, $\int l_{1} \times K X=0$ iff $K k\left(l_{1}\right) \in$ sym, i.e. $K \in T_{I} S_{A_{1}}$.

3.10. LEMMA. Suppose $u_{1}, \ldots, u_{n-1}$ satisfy $\left(\mathrm{I}_{1}\right), \ldots,(\mathrm{I})_{n-1}$. Then, there exist $\phi=1+\lambda u_{1}+\lambda^{2} u_{2}+\cdots+\lambda^{n-1} u_{n-1}+\cdots$ such that $J(\phi)=1$.

PROOF. Let $\pi$ be a projection of $\mathcal{U}$ onto the tangent space $\mathcal{V}=\{u \in \mathcal{U} \mid \operatorname{tr} \nabla u=$ $0\}$ of $C_{\text {vol }}$ at $I$. By the inverse mapping theorem there exists unique $\phi=1+\lambda u_{1}^{*}+$ $\lambda^{2} u_{2}^{*}+\cdots$ in $C_{\text {vol }}$ such that

$$
\pi\left(\lambda u_{1}^{*}+\lambda^{2} u_{2}^{*}+\cdots\right)=\pi\left(\lambda u_{1}+\lambda^{2} u_{2}+\cdots+\lambda^{n-1} u_{n-1}\right) .
$$

Thus, $\pi\left(u_{1}^{*}\right)=\pi\left(u_{1}\right), \ldots, \pi\left(u_{n-1}^{*}\right)=\pi\left(u_{n-1}\right)$. Using $\left(\mathrm{I}_{1}\right), \ldots,\left(\mathrm{I}_{n-1}\right)$, we obtain $u_{1}^{*}=u_{1}, \ldots, u_{n-1}^{*}=u_{n-1}$. Q.E.D.

3.11. Lemma. Let $l_{1} \in \mathcal{L}_{e}$. Suppose $\left(p_{1}, u_{1}\right), \ldots,\left(p_{n-1}, u_{n-1}\right)$ satisfies $\left(\mathrm{C}_{1}\right)$, $\left(\mathrm{L}_{1}\right),\left(\mathrm{I}_{1}\right), \ldots,\left(\mathrm{C}_{n-2}\right),\left(\mathrm{L}_{n-2}\right),\left(\mathrm{I}_{n-2}\right),\left(\mathrm{L}_{n-1}\right)$ and $\left(\mathrm{I}_{n-1}\right)$. Then, $\left(\mathrm{C}_{n-1}\right)$ is a solvability condition of $\left(\mathrm{L}_{n}\right)$ and $\left(\mathrm{I}_{n}\right)$ for $p_{n}, u_{n}$.

ProOF. Choose $\phi=1+\lambda u_{1}+\lambda^{2} u_{2}+\cdots+\lambda^{n-1} u_{n-1}+\lambda^{n} u_{n}^{*}+\cdots$ by Lemma 3.10. Set

$$
\begin{gathered}
\Phi\left(\lambda p_{1}+\lambda^{2} p_{2}+\cdots+\lambda^{n-1} p_{n-1}, I+\lambda u_{1}+\lambda^{2} u_{2}+\cdots+\lambda^{n-1} u_{n-1}+\lambda^{n} u_{n}^{*}+\cdots\right) \\
=\lambda l_{1}+\lambda^{2} l_{2}+\cdots+\lambda^{n-1} l_{n-1}+\lambda^{n} l_{n}^{*}+\cdots
\end{gathered}
$$


So $u_{n}^{*}$ satisfies $\left(\mathrm{I}_{n}^{*}\right),\left(\mathrm{L}_{n}^{*}\right): L\left(0, u_{n}^{*}\right)+\mathcal{K}\left(\left(p_{1}, u_{1}\right), \ldots,\left(p_{n-1}, u_{n-1}\right)\right)=l_{n}^{*}$, and $\left(\mathrm{C}_{n-1}^{*}\right)$ : $\int l_{1} \times u_{n-1}+\cdots+\int l_{n}^{*} \times I=0$. One needs to find $p_{n}, u_{n}=u_{n}^{*}+v$ such that $\operatorname{tr} \nabla v=0$ and $L\left(p_{n}, v\right)=l_{n}-l_{n}^{*}$. Since $\int l_{1} \times u_{n-1}+\cdots+\int l_{n} \times I=0, \int l_{1} \times u_{n-1}+\cdots+$ $\int l_{n}^{*} \times I=0 \int\left(l_{n}-l_{n}^{*}\right) \times I=0$ or $l_{n}-l_{n}^{*} \in \mathcal{L}_{e}$. Consequently, such $p_{n}, u_{n}$ can be found by Proposition 1.2. Q.E.D.

Now, we are ready to present the Signorini Scheme for incompressible materials.

3.12. THEOREM. Let $l_{1} \in \mathcal{L}_{e}$ have no axis of equilibria. Suppose $\left(p_{1}, u_{1}\right), \ldots$, $\left(p_{n-1}, u_{n-1}\right)(n \geq 1)$ satisfies $\left(\mathrm{C}_{1}\right),\left(\mathrm{L}_{1}\right),\left(\mathrm{I}_{1}\right), \ldots,\left(\mathrm{C}_{n-1}\right),\left(\mathrm{L}_{n-1}\right)$ and $\left(\mathrm{I}_{n-1}\right)$. Then $\left(\mathrm{C}_{n}\right),\left(\mathrm{L}_{n}\right)$ and $\left(\mathrm{I}_{n}\right)$ define $p_{n}, u_{n}$.

ProOF. By Lemma 3.11, there exists $\left(p_{n}, \tilde{u}_{n}\right)$ which satisfies $\left(\mathrm{L}_{n}\right),\left(\mathrm{I}_{n}\right)$. One needs to find $K \in$ skew, $u_{n}=\tilde{u}_{n}+K X$, so that $\left(\mathrm{C}_{n}\right)$ holds, i.e.

$$
\int l_{1} \times u_{n}+\cdots+\int l_{n+1} \times I=0,
$$

or

$$
\int l_{1} \times K X=-\left(\int l_{1} \times \tilde{u}_{n}+\cdots+\int l_{n+1} \times I\right) .
$$

For $l_{1}$ has no axis of equilibria, $T_{I} S_{A_{1}}=\{0\}$, by Lemma 3.9 , the map $K \rightarrow$ $\int l_{1} \times K X$ is an isomorphism. Therefore, such a $K \in$ skew can be found and indeed, it must be unique. Q.E.D.

(C) A second order sufficient condition, and linearization stability. Now we will extend Theorem 3.12 so that the load $l_{1}$ may possess an axis of equilibria (i.e. $\left.T_{I} S_{A_{1}} \neq\{0\}\right)$.

3.13. THEOREM. Suppose $2\left\langle l_{2}, Q^{T} I\right\rangle+\left\langle c\left(\nabla u_{Q}\right), \nabla u_{Q}\right\rangle$ has a nondegenerate critical point at $I$ restricted to $S_{A_{1}}$. Then, there exist $p(\lambda), \phi(\lambda) \in C_{\mathrm{vol}}$ such that $\Phi(p(\lambda), \phi(\lambda))=l(\lambda), p(\lambda) \rightarrow 0, \phi(\lambda) \rightarrow I$, as $\lambda \rightarrow 0$.

ProOF. It suffices to observe, by Proposition 2.16,

$$
\tilde{f}=\text { constant }-\lambda\left\langle l_{2}, Q^{T} 1\right\rangle-\frac{\lambda}{2}\left\langle c\left(\nabla u_{Q}\right), \nabla u_{Q}\right\rangle+O\left(\lambda^{2}\right) . \quad \text { Q.E.D. }
$$

The above theorem enables us to relate the linear elasticity theory (cf. [9]) to that of nonlinear elasticity theory in a satisfactory and expected way (cf. $[\mathbf{1 5}, \mathbf{1 6}])$. The appropriate notion one may introduce is the following

3.14. Definition (CF. [6]). Given $l_{1} \in \mathcal{L}_{e}$. The load $l_{1}$ is said to be linearization stable in $\mathcal{L}_{e}$ if there exist $p(\lambda), \phi(\lambda) \in \mathcal{C}_{\mathrm{vol}}, \Phi(p(\lambda), \phi(\lambda))=l(\lambda), p(\lambda) \rightarrow 0, \phi(\lambda) \rightarrow$ $I$ as $\lambda \rightarrow 0$ for some $l(\lambda) \in \mathcal{L}_{e}, l^{\prime}(0)=l_{1}$. If $l_{1} \in \mathcal{L}_{e}$ is linearization stable in $\mathcal{L}_{e}$, then there exist $p_{1}, u_{1}$, such that $L\left(p_{1}, u_{1}\right)=l_{1}, \operatorname{tr} \nabla u_{1}=0$ with $\int l_{1} \times u_{1}=0$. Conversely, we have

3.15. THEOREM. A load $l_{1} \in \mathcal{L}_{e}$ is linearization stable in $\mathcal{L}_{e}$ if there exist $p_{1}, u_{1}$ such that $L\left(p_{1}, u_{1}\right)=l_{1}, \operatorname{tr} \nabla u_{1}=0, \int l_{1} \times u_{1}=0$.

In other words, $\left(\mathrm{C}_{1}\right),\left(\mathrm{I}_{1}\right),\left(\mathrm{L}_{1}\right)$ are the only obstructions for the solvability of the equation $\Phi(p(\lambda), \phi(\lambda))=l(\lambda)$, with $l^{\prime}(0)$ given.

Proof. By Theorem 3.6, $\left\langle c\left(\nabla u_{Q}\right), \nabla u_{Q}\right\rangle$ has a critical point at $Q=I$. Take $l_{2} \in \mathcal{L}_{e}$ so that $2\left\langle l_{2}, Q^{T} I\right\rangle+\left\langle c\left(\nabla u_{Q}\right), \nabla u_{Q}\right\rangle$ is nondegenerate at $I$ along $S_{A_{1}}$. By 
Theorem 3.13 there exist $p(\lambda), \phi(\lambda) \in \mathcal{C}_{\mathrm{vol}}, \Phi(p(\lambda), \phi(\lambda))=\lambda l_{1}+\lambda^{2} l_{2}, p(\lambda) \rightarrow 0$, $\phi(\lambda) \rightarrow I$ as $\lambda \rightarrow 0$. Q.E.D.

(D) A generalized Signorini Scheme. Here, we present a scheme for the solution found in Theorem 3.13. This scheme extends the Signorini Scheme given by Theorem 3.12 .

3.16. THEOREM. Let the hypothesis in Theorem 3.13 be fulfilled. Suppose $\left(p_{1}, u_{1}\right), \ldots,\left(p_{n-2}, u_{n-2}\right)(n \geq 1)$ and $p_{n-1}, u_{n-1} \bmod K X, K \in T_{I} S_{A_{1}}$, are determined through $\left(\mathrm{C}_{1}\right),\left(\mathrm{I}_{1}\right),\left(\mathrm{L}_{1}\right), \ldots,\left(\mathrm{C}_{n-1}\right),\left(\mathrm{I}_{n-1}\right),\left(\mathrm{L}_{n-1}\right)$. Then, equations $\left(\mathrm{C}_{n}\right),\left(\mathrm{I}_{n}\right)$ and $\left(\mathrm{L}_{n}\right)$ define $u_{n-1}$ and $p_{n}, u_{n} \bmod K X, K \in T_{I} S_{A_{1}}$.

Our proof consists of brute force computations. Basically, Lemmas 3.17-3.19 are collections of relevant facts similar to that in $[\mathbf{1 1}]$.

3.17. Lemma. (a) $2\left\langle l_{2}, Q^{T} X\right\rangle+\left\langle c\left(\nabla u_{Q}\right), \nabla u_{Q}\right\rangle$ has a critical point on $S_{A_{1}}$ at $Q=I$ iff $\left\langle l_{2}, K^{T} X\right\rangle+\left\langle c\left(\nabla u_{Q}\right), \nabla u_{Q}\right\rangle=0$ for all $K \in T_{I} S_{A_{1}}$.

(b) The Hessian is $\left\langle l_{2}, K^{2} X\right\rangle+\left\langle c\left(\nabla u_{K}\right), \nabla u_{K}\right\rangle+\left\langle c\left(\nabla u_{I}\right), \nabla u_{K^{2}}\right\rangle$ for all $K \in$ $T_{I} S_{A_{1}}$.

Expand $P=P_{1}+P_{2}+\cdots$ and $\Phi=\Phi_{1}+\Phi_{2}+\cdots$ as formal series at $(0, I)$ and $\left(0, I_{B}\right)$ respectively. Thus, $\Phi_{1}=L$.

$$
\begin{aligned}
P & =-J q F^{-T}+P^{*} \\
& =-(1+\operatorname{tr} H+\cdots) q\left(1-H^{T}+\cdots\right)+\frac{\partial P^{*}}{\partial F}(H)+\frac{1}{2} \frac{\partial^{2} P^{*}}{\partial F^{2}}(H)^{2}+\cdots \\
& =\left[-q+\frac{\partial P^{*}}{\partial F}(H)\right]+\left[q\left(H^{T}-\operatorname{tr} H\right)+\frac{1}{2} \frac{\partial^{2} P^{*}}{\partial F^{2}}(H)^{2}\right]+\cdots, \quad P^{*}=\frac{\partial W}{\partial F} .
\end{aligned}
$$

Hence, $P_{1}=-q+\left(\partial P^{*} / \partial F\right)(H)$ and $P_{2}=q\left(H^{T}-\operatorname{tr} H\right)+\frac{1}{2}\left(\partial^{2} P^{*} / \partial F^{2}\right)(H)^{2}$.

Applying the divergence theorem, we get

3.18. LEMMA. (a) $\langle L(q, u), w\rangle=\left\langle P_{1}(q, \nabla u), \nabla w\right\rangle$,

(b) $\left\langle\Phi_{2}(q, u)^{2}, w\right\rangle=\left\langle P_{2}(q, \nabla u)^{2}, \nabla w\right\rangle$.

Now, set $W=W(D), D=\left(F^{T} F-1\right) / 2$. Thus $P^{*}=\partial W / \partial F, S^{*}=\partial W / \partial D$ and $P^{*}(F)=F S^{*}(D)$. AT $F=I$, computations show

3.19. LEMMA. (a) $\left(\partial P^{*} / \partial F\right)(H)=\left(\partial S^{*} / \partial D\right)(H)$,

(b)

$$
\frac{\partial^{2} P^{*}}{\partial F^{2}}(H, K)=H \frac{\partial S^{*}}{\partial D}(K)+K \frac{\partial S^{*}}{\partial D}(H)+\frac{\partial S^{*}}{\partial D}\left(K^{T} H\right)+\frac{\partial^{2} S^{*}}{\partial D^{2}}(H, K) .
$$

3.20. LEMMA. (a) $\Phi_{2}(0, K X)^{2}=L\left(0, \frac{1}{2} K^{T} K X\right)$.

(b) $2 \Phi_{2}\left(\left(p_{1}, u_{1}\right),(0, K X)\right)=K l_{1}+L\left(0, K^{T} u_{1}\right)$ for $K \in T_{I} S_{A_{1}}$.

PROOF. (a)

$$
\begin{aligned}
\left\langle 2 \Phi_{2}(0, K X)^{2}, w\right\rangle & =\left\langle\frac{\partial S^{*}}{\partial D}\left(K^{T} K\right), \nabla w\right\rangle \quad(\text { by Lemmas 3.18(b) and 3.19(b)) } \\
& =\left\langle L\left(0, K^{T} K X\right), w\right\rangle \quad(\text { by Lemmas 3.18(a) and 3.19(a)) }
\end{aligned}
$$

for all $w \in \mathcal{U}$. 
(b)

$$
\begin{aligned}
\left\langle 2 \Phi_{2}\left(\left(p_{1}, u_{1}\right),(0, K X)\right), w\right\rangle & =\left\langle 2 \Phi_{2}((p, 0),(0, K X))+2 \Phi_{2}\left(\left(0, u_{1}\right)(0, K X)\right), w\right\rangle \\
& =\left\langle p_{1} K^{T}+K \frac{\partial S^{*}}{\partial D}(H)+\frac{\partial S^{*}}{\partial D}\left(K^{T} H\right), \nabla w\right\rangle \\
& \quad \text { by Lemmas 3.18(b) and 3.19(b)) } \\
& =\left\langle K\left(-p_{1}+\frac{\partial S^{*}}{\partial D}(H)\right)+\frac{\partial S^{*}}{\partial D}\left(K^{T} H\right), \nabla w\right\rangle \\
& =\left\langle K l_{1}+L\left(0, K^{T} u_{1}\right), w\right\rangle, \quad \text { for all } w \in \mathcal{U} . \quad \text { Q.E.D. }
\end{aligned}
$$

3.21. Lemma. $\left\langle W l_{1}, K^{T} u_{1}\right\rangle-\left\langle W l_{2}, K X\right\rangle$ is symmetric in $W$ and $K$, where $W, K \in T_{I} S_{A_{1}}$.

Proof. $\int l_{1} \times u_{1}+\int l_{2} \times I=0$ means $k\left(l_{1}, u_{1}\right)+k\left(l_{2}, I\right) \in$ sym. Thus, $\left\langle\tilde{K}^{T}, k\left(l_{1}, u_{1}\right)\right\rangle+\left\langle\tilde{K}^{T}, k\left(l_{2}, I\right)\right\rangle=0$ for all $\tilde{K} \in$ skew, or $\left\langle\tilde{K} l_{1}, u_{1}\right\rangle+\left\langle\tilde{K} l_{2}, X\right\rangle=0$.

Let $\tilde{K}=W K-K W$. Then one obtains

$$
\left\langle W K l_{1}, u_{1}\right\rangle+\left\langle W K l_{2}, X\right\rangle=\left\langle K W l_{1}, u_{1}\right\rangle+\left\langle K W l_{2}, X\right\rangle,
$$

or

$$
\left\langle K l_{1}, W^{T} u_{1}\right\rangle-\left\langle K l_{2}, W X\right\rangle=\left\langle W l_{1}, K^{T} u_{1}\right\rangle-\left\langle W l_{2}, K X\right\rangle \text {. Q.E.D. }
$$

Now, we are ready to prove Theorem 3.16 in steps $(\alpha),(\beta),(\gamma)$.

$(\alpha)$ Let $n>2$. One needs to show that there exists a unique $K \in T_{I} S_{A_{1}}$ such that $u_{n-1}=u_{n-1}^{*}+K X\left(u_{n-1}^{*}\right.$ given by hypothesis $)$, and a corresponding $u_{n}, p_{n}$ obtained by Lemma 3.11 , solve $\left(\mathrm{L}_{n}\right),\left(\mathrm{I}_{n}\right)$ and $\left(\mathrm{C}_{n}\right)$.

For each $K \in T_{I} S_{A_{1}}$, from $\left(\mathrm{I}_{n}\right),\left(\mathrm{L}_{n}\right)$ for $p_{n}, u_{n}$ and $\left(\mathrm{I}_{n}^{*}\right),\left(\mathrm{L}_{n}^{*}\right)$ for $p_{n}^{*}, u_{n}^{*}$ (given by Lemma 3.11)

$$
\begin{gathered}
L\left(p_{n}-p_{n}^{*}, u_{n}-u_{n}^{*}\right)+2 \Phi_{2}\left(\left(p_{1}, u_{1}\right),(0, K X)\right)=0, \\
L\left(p_{n}-p_{n}^{*}+p_{K}, u_{n}-u_{n}^{*}+u_{K}+K^{T} u_{1}\right)=0 \quad \text { (by Lemma 3.20(b)). }
\end{gathered}
$$

For, $\operatorname{tr}\left(\nabla u_{n}-\nabla u_{n}^{*}+\nabla u_{K}+K^{T} H\right)=\operatorname{tr}\left(\nabla u_{n}-\nabla u_{n}^{*}+K^{T} H\right)=0$ by $\left(\mathrm{I}_{n}\right),\left(\mathrm{I}_{n}^{*}\right)$. Thus, $u_{n}-u_{n}^{*}+u_{K}+K^{T} u_{1}+\tilde{K} X=0$ and $p_{n}-p_{n}^{*}+p_{K}=0$ for some $\tilde{K} \in$ skew. Putting in $\left(\mathrm{C}_{n}\right)$, one has

$$
\begin{aligned}
\int l_{1} \times\left(u_{n}^{*}-u_{K}-K^{T} u_{1}-\tilde{K} X\right) & +\int l_{2} \times\left(u_{n-1}^{*}+K X\right) \\
& +\int l_{3} \times u_{n-2}+\cdots+\int l_{n+1} \times I=0 .
\end{aligned}
$$

or

$$
-k\left(l_{1}, u_{K}+K^{T} u_{1}+\tilde{K} X\right)+k\left(l_{2}, K X\right)+M \in \mathrm{sym},
$$

with $M=k\left(l_{1}, u_{n}^{*}\right)+k\left(l_{2}, u_{n-1}^{*}\right)+k\left(l_{3}, u_{n-2}\right)+\cdots+k\left(l_{n+1}, I\right)$. Since, $\left\langle W, k\left(l_{1}, \tilde{K} X\right)\right\rangle$ $=\left\langle-W l_{1}, \tilde{K} X\right\rangle=\left\langle-W A_{1}, \tilde{K}\right\rangle=0$ for $W \in T_{I} S_{A_{1}}$

(2) $\left\langle W,-k\left(l_{1}, u_{k}+K^{T} u_{1}\right)\right\rangle+\left\langle W, k\left(l_{2}, K X\right)\right\rangle+\langle W, M\rangle=0$ for all $W \in T_{I} S_{A_{1}}$.

Now, $\left\langle W,-k\left(l_{1}, u_{K}+K^{T} u_{1}\right)\right\rangle+\left\langle W, k\left(l_{2}, K X\right)\right\rangle=\left\langle W l_{1}, u_{K}\right\rangle+\left\langle W l_{1}, K^{T} u_{1}\right\rangle-$ $\left\langle W l_{2}, K X\right\rangle$ is a nondegenerate form by Lemmas 3.21 and 3.20(b). 
Thus, there exists a unique $K \in T_{I} S_{A_{1}}$ such that equation (2) holds. Thus, one gets the uniqueness part of $K$ in the theorem. To obtain the existence part, one seeks $\tilde{K} \in$ skew so that

$$
u_{n-1}=u_{n-1}^{*}+K X, \quad u_{n}=u_{n}^{*}-u_{K}-K^{T} u_{1}-\tilde{K} X, \quad p_{n}=p_{n}^{*}-p_{K}
$$

satisfies $\left(\mathrm{C}_{n}\right)$. (Conditions $\left(\mathrm{I}_{n}\right)$ and $\left(\mathrm{L}_{n}\right)$ are fulfilled automatically.) Consider the equation for $\tilde{K}$ :

$$
\begin{aligned}
k\left(l_{1}, \tilde{K} X\right) & =-k\left(l_{1}, u_{k}+K^{T} u_{1}\right)+k\left\langle l_{2}, K X\right\rangle+M \quad \bmod \text { sym } \\
& =N \quad \text { mod sym. }
\end{aligned}
$$

From $\left\langle\tilde{K}, k\left(W^{T} l_{1}\right)\right\rangle=\left\langle k\left(l_{1}, \tilde{K}(X)\right), W\right\rangle$, the solvability condition for $\tilde{K}$ is $\langle W, N\rangle$ $=0$ for all $W \in T_{I} S_{A_{1}}$, which is precisely the equation (2).

( $\beta$ ) The proof for $n=2$ is basically the same as in $(\alpha)$, where one needs Lemma $3.20(\mathrm{a})$.

Indeed, one has

$$
L\left(p_{2}-p_{2}^{*}, u_{2}-u_{2}^{*}\right)+2 \Phi_{2}\left(\left(p_{1}, u_{1}\right),(0, K X)\right)-\Phi_{2}(0, K X)^{2}=0
$$

and

$$
L\left(p_{2}-p_{2}^{*}+p_{K}, u_{2}-u_{2}^{*}+u_{K}+K^{T} u_{1}-\frac{1}{2} K^{T} K X\right)=0
$$

by Lemma 3.20. For, $\operatorname{tr}\left(\nabla u_{2}-\nabla u_{2}^{*}+\nabla u_{K}+K^{T} H-\frac{1}{2} K^{T} K\right)=0$ by $\left(\mathrm{I}_{2}\right),\left(\mathrm{I}_{2}^{*}\right)$. Thus $u_{2}-u_{2}^{*}+u_{K}+K^{T} u_{1}-\frac{1}{2} K^{T} K X+\tilde{K} X=0$ and $p_{2}-p_{2}^{*}+p_{K}=0$ for some $\tilde{K} \in$ skew. Putting in $\left(\mathrm{C}_{2}\right)$, for $\int l_{1} \times K^{T} K X=0$ or $k\left(l_{1}, K^{T} K X\right) \in$ sym (notice $\left.K \in T_{I} S_{A_{1}}\right)$. One has the same equations for $K, \tilde{K}$ as equation (1).

The rest of the proof is the same as that in $(\alpha)$.

$(\gamma)$ When $n=1$ this is Theorem 3.6. Q.E.D.

\section{REFERENCES}

1. J. Ball and D. Schaeffer, Bifurcation and stability of homogeneous equilibrium configurations of an elasticity body under dead-load tractions, preprint, 1982.

2. D. Chillingworth, J. Marsden and Y. H. Wan, Symmetry and bifurcation in three dimensional elasticity. Part I, Arch. Rational Mech. Anal. 80 (1982), 295-333.

3. __ Symmetry and bifurcation in three dimensional elasticity, Part II, Arch. Rational Mech. Anal. 83 (1983), 363-395.

4. D. Ebin and J. Marsden, Groups of diffeomorphisms and the motion of an incompressible fluid, Ann. of Math. (2) 92 (1970), 102-163.

5. G. Fichera, Existence theorems in elasticity, Handbuch der Physik, Bd. VIa/2, Springer-Verlag, Berlin and New York, 1972, pp. 347-389.

6. A. Fischer and J. Marsden, Linearization stability of nonlinear partial differential equations, Proc. Sympos. Pure. Math., vol. 27, Amer. Math. Soc., Providence, R.I., 1975, pp. 219-263.

7. A. E. Green and E. B. Spratt, Second-order effects in the deformation of elastic bodies, Proc. Roy. Soc. London Ser. A 224, 347-361.

8. G. Grioli, Mathematical theory of elastic equilibrium, Ergeb. Math. Grenzgeb., Band 7, SpringerVerlag, Berlin and New York, 1962.

9. M. Gurtin, The linear theory of elasticity, Handbuch der Physik, vol. VIa/2, Springer-Verlag, Berlin, 1972, pp. 1-295.

10. J. Marsden and T. Hughes, The mathematical foundations of elasticity, Prentice-Hall, Englewood Clifs, N.J., 1982.

11. J. Marsden and Y. Wan, Linearization stability and Signorini series for the traction problem in elastostatics, preprint, 1982. 
12. A. Signorini, Sulle deformazioni termoelastiche finite, Proc. 3rd Internat. Congr. Appl. Mech., vol. 2,1930 , pp. 80-89.

13. F. Stoppelli, Sulle esistenza di soluzioni delli equazioni del' elastostatica isoterma rel case di sollectizioni detate di assi di equilibria, Richerche Mat. 6 (1957), 241-287; 7 (1958), 71-101, 138-152.

14. C. Tolotti, Orientamenti principali di un corpo elastico rispetto alla sua sollecitazione totale, Mem. Accad. Italia Cl. Sci. Math. 13 (1943), 1139-1162.

15. C. Truesdell and W. Noll, The nonlinear field theories of mechanics, Handbuch der Physik III/3 (S. Flugge, ed.), Springer-Verlag, Berlin and New York, 1965.

16. C. Wang and C. Truesdell, Introduction to rational elasticity, Noordhoff, Groningen, 1973.

Department of Mathematics, State University of NeW York, Buffalo, NeW YORK 14214 